\title{
Características del enfermero y enfermera que ejerce la enseñanza universitaria ${ }^{1}$
}

Institución: Universidad de Costa Rica

Johnny Alvarado Sojo ${ }^{2}$

\section{COMO CITAR}

Alvarado, J. (2016). Características del enfermero y enfermera que ejerce la enseñanza a nivel universitario. Rev. Enfermería Actual en Costa Rica, 31, 1-14. DOI: http://dx.doi.org/10.15517/revenf.v0i31.25632

\section{RESUMEN}

Introducción. El siguiente artículo es producto de una investigación que tuvo como objetivo analizar las características que requiere el enfermero o la enfermera para ejercer la docencia universitaria.

Método. Se realizó una revisión de literatura, donde se utilizaron diversos artículos completos, indexados en bases de datos EBSCO y Directory of Open Access Journals, recuperando 65, de los cuales solo siete se relacionaron directamente con características o cualidades del docente.

Resultado. Se agruparon en dos categorías: docencia universitaria e innovación en docencia, presentando posibles requerimientos que se necesitan para ser docente universitario, pero en muy pocos casos se hizo mención específica a la Enfermería.

Conclusión. La mayoría de los autores coinciden que la selección de los docentes universitarios y su proceso de desarrollo académico no puede limitarse a lo disciplinar, deberá asumir lo personal y lo pedagógico, de hecho lo pedagógico no existe si no es al interior de una disciplina. Además, de ser experto en el campo que enseña es desarrollador de investigación, acción social.

Palabras clave: competencias-docentes, educación-en-enfermería, enseñanza-universitaria.

\footnotetext{
${ }^{1}$ Fecha de recepción: 04 marzo 2015

Fecha de aceptación: 14 junio del 2016

${ }^{2}$ Enfermero. Máster en Salud Laboral. Docente de la Universidad de Costa Rica. Costa Rica. Correo electrónico:

Johnny.alvaradosojo@ucr.ac.cr
} 


\section{Characteristics of nurse practicing university education ${ }^{1}$}

Institution: University of Costa Rica.

\section{CITED}

Alvarado, J. (2016). Characteristics of nurse practicing university education. Rev. Enfermería Actual en Costa Rica, 31, 1-14. DOI: http://dx.doi.org/10.15517/revenf.v0i31.25632

Introduction. The following article is the result of an investigation that aimed to analyze the characteristics required by the nurse to exert university teaching.

Method. A literature review, where several full articles were used, indexed in EBSCO databases and Directory of Open Access Journals data, recovering 65, of which only seven were directly related characteristics or qualities of teachers was conducted.

Result. They were grouped into two categories: university teaching and innovation in teaching, presenting possible requirements needed to be a university teacher, but in very few cases mention was made specific to nursing.

Conclusion. Most authors agree that the selection of university teachers and academic development process can not be limited to the discipline, must assume personal and educational, pedagogical fact does not exist if not within a discipline. In addition to being expert in the field teaching is to be developer of research, social action.

Key words: education-in-nursing, skills-teaching, teaching-college.

\footnotetext{
${ }^{1}$ Date Received: April 7, 2016

Date of acceptance: June 14, 2016

${ }^{2}$ Nurse. Master in Occupational Health. Professor at the University of Costa Rica. Costa Rica. E-mail: Johnny.alvaradosojo@ucr.ac.cr
} 


\section{Características enfermeiro e enfermeira praticando o ensino universitário ${ }^{1}$}

Johnny Alvarado Sojo ${ }^{2}$

Instituição: Universidade da Costa Rica

\section{CITAÇÃO}

Alvarado, J. (2016 Características enfermeiro e enfermeira praticando o ensino universitário. Rev. Enfermería Actual en Costa Rica, 31, 1-14. DOI: http://dx.doi.org/10.15517/revenf.v0i31.25632

\section{RESUMO}

Introdução. O artigo a seguir é o resultado de uma investigação que teve como objetivo analisar as características exigidas pela enfermeira ou enfermeiro para exercer a docência universitária.

Método. Uma revisão da literatura, em que foram utilizados vários artigos completos, indexados em bases de dados da EBSCO e Directory of Open Access Journals de dados, recuperação de 65, dos quais características ou qualidades de professores só sete foram diretamente relacionados foi realizado.

Resultado. Eles foram agrupados em duas categorias: o ensino a nível universitário e inovação no ensino, apresentando possíveis requisitos necessários para ser um professor universitário, mas em muito poucos casos menção foi feita específico para a enfermagem.

Conclusão. A maioria dos autores concorda que a selecção dos professores universitários e processo de desenvolvimento acadêmico não pode ser limitada à disciplina, deve assumir pessoal e educacional, fato pedagógica não existe se não for dentro de uma disciplina. Além de ser especialista no ensino de campo é a pesquisa desenvolvedor, ação social.

Palavras-chave: Habilidades-de-ensino, educação-in-enfermagem, ensino-universitário.

\footnotetext{
${ }^{1}$ Data de recepção: 07 de abril de 2016

Data de aceitação: 14 de junho de 2016

${ }^{2}$ Enfermeiro. Mestre em Saúde Ocupacional. Professor na Universidade de Costa Rica. Costa Rica. Correio eletrônico:

Johnny.alvaradosojo@ucr.ac.cr
} 


\section{Revista Electrónica Enfermeria Actual en costa Rica}

\section{INTRODUCCIÓN}

En el proceso enseñanza - aprendizaje, se relacionan diversos factores que influyen en el cómo se enseña y el cómo se aprende, entre estos y desde la perspectiva docente se puede hacer referencia a la experiencia, las cualidades, particularidades o las características que éste posea.

Respecto de las características o particularidades del docente, Bain (2006), hace mención a una serie de preguntas, que llevan a la reflexión respecto de la definición de los mejores docentes, algunas de estas preguntas son: “¿qué saben y entienden los mejores profesores?”(p.26), “¿cómo preparan su docencia?” “¿qué esperan de sus estudiantes?”(p.28) “¿qué hacen cuando enseñan?” “¿cómo tratan a sus estudiantes?(p.29) y “¿cómo comprueban su progreso y evalúan sus necesidades?”(p.30).

Debido a lo anterior, surge la interrogante de valorar el establecimiento de ciertas características que debe tener un docente universitario y colocarlas en un perfil que lo describa.

Canalejas-Pérez, Martínez-Martín, Pineda-Ginés, Vera-Cortés, Soto-González, Martín-Marino y CidGalán (2005), comprobó por medio de un estudio observacional, exploratorio y transversal, que si el docente logra obtener ciertas cualidades con un perfil teórico-reflexivo "el alumno va a tener más facilidad para aprender de cualquier forma y aprovechar las oportunidades de aprendizaje que se encuentre a lo largo de su vida" (p. 87), en comparación que si llegaran a tener un perfil más activo-pragmático, para lo cual los docentes deben tener un conocimiento pedagógico y metodológico muy claro para poder hacer esa relación entre lo que se enseña y se práctica.

Por lo tanto, la formación del profesorado llega a tener importancia, donde dicha formación debe ser congruentes con las políticas institucionales, tal como menciona Preciado (2008), éstas políticas tienen un gran impacto en el quehacer docente, ya que todo docente universitario al no contar con una formación más allá de la formación profesional muy difícilmente va a lograr cumplir con sus objetivos. Relacionado con esto Francis (2010) hace énfasis en que la selección de los docentes universitarios y su proceso de desarrollo académico no puede limitarse a lo disciplinar, deberá asumir lo personal y lo pedagógico (p.44)

De esta manera, el docente universitario debe poseer una variedad de características y conocimientos académicos, para desarrollar las estrategias necesarias que garanticen un adecuado proceso de enseñanza y aprendizaje.

En el contexto de la Universidad de Costa Rica, se puede encontrar registrado en la sesión ordinaria $\mathrm{n}^{\circ} 4926$ del Consejo Universitario (2004), en el artículo 6 el "perfil de la competencias para el profesorado de la Universidad de Costa Rica", el cual tiene como objetivo general: "Dictaminar sobre el perfil de competencias deseado para el profesorado de la Universidad de Costa Rica en relación a las exigencias gestadas por la dinámica mundial de las instituciones públicas de Educación superior" (p.34).

Específicamente en el campo de la Enfermería, al hablar de un perfil de competencias, se debe considerar la definición que plantea Medina (1999) mencionando que la enfermería "es una disciplina científica que posee un objeto particular de estudio y un cuerpo propio de saberes edificados a partir de él" (p.29), por lo tanto, la 


\section{Revista Electrónica Enfermeria Actual en costa Rica}

formación que debe llevar el estudiantado de enfermería debe ser congruente con su objeto de estudio y considerar un enseñanza científica, tecnológica y humanística.

A finales del siglo XIX, cuando se crea la primer "escuela de parteras", como parte de la instauración de los servicios de atención en salud en la sociedad costarricense, convirtiéndose más adelante en la "Escuela de Obstetricia de Costa Rica", todas las estudiantes de enfermería debían estar al cuidado de profesores cuya profesión era la medicina nombrados por la Facultad de Medicina, hoy en día Colegio de Médicos (Mora-Quirós y Caravaca-Morera, 2014). Esto continúo a inicios del siglo XX, cuando se crea la Escuela de Enfermería, ubicada en el hospital San Juan de Dios, la cual trabajaba a parte de la Escuela de Obstetricia, fucionándose tiempo después.

Según Mora-Quirós y Caravaca-Morera (2014) es "hasta el año 1930 que se contrata por primera vez una enfermera Miss Dorothy Anderson, para impartir educación en servicio a las asistentes sanitarias escolares y actualizar algunos principios básicos de enfermería y protección materno-infantil” (p. 288).

En la actualidad la formación de enfermeras y enfermeros, al igual que en otras áreas de salud, no necesariamente ha estado a cargo de profesionales en docencia directamente, sino de los mismos profesionales en enfermería, que de una manera autodidacta han tratado de ejercer la docencia.

Debido a lo anterior, se hace necesario evidenciar las características que debe tener un docente de enfermería de la Universidad de Costa Rica con la finalidad de mejorar los procesos de enseñanza y aprendizaje de la enfermería. Por ello, esta revisión de literatura se planteó como objetivo, analizar las evidencias científicas disponibles referentes a las características que requiere el enfermero o la enfermera para ejercer la docencia.

\section{MATERIALES Y MÉTODOS}

Esta investigación se basa en una revisión de literatura que según los estudios de Ganong (1987), Broome (2000) y Whittemore y Knalf (2005), es un método de revisión específico que permite incluir diversas líneas de investigación, tanto cuantitativas como cualitativas. Se implementaron seis etapas:

\section{Identificación del problema.}

En este tópico se planteó la siguiente pregunta: ¿Cuáles son las características que debe tener un enfermero o enfermera que ejerce la docencia universitaria?

\section{Selección de la muestra por medio del establecimiento de criterios de exclusión e inclusión.}

Los criterios considerados para la inclusión de las publicaciones de la presente revisión integradora fueron: artículos completos disponibles electrónicamente que abordaran el tema de características que deben poseer los docentes para ejercer la enseñanza, específicamente a nivel universitario; artículos indexados, publicados en idiomas portugués, castellanos e inglés, en el periodo de marzo a junio del 2014, y que presenten con claridad el diseño del estudio y la población utilizada para la investigación. Fueron recuperados 65 artículos, de los cuales solo 7 tenían relación directa con los criterios de inclusión que fueron descritos anteriormente. La procedencia de los artículos revisados fueron: Costa Rica, España, y Chile. 


\section{Revista Electrónica Enfermeria Actual en costa Rica}

\section{Definición de la información que será extraída de los artículos seleccionados.}

Para la búsqueda de los artículos científicos, se consultó las bases de datos EBSCO y Directory of Open Access Journals, por medio de los descriptores en el Thesauro de la UNESCO: enseñanza superior, profesionales de la educación, y el descriptor en ciencias de la salud DeSc: educación en enfermería. La recuperación de las publicaciones se llevo a cabo en los meses de marzo a junio del 2014.

\section{Análisis de la información.}

El análisis y síntesis de los datos se efectuó con una lectura minuciosa, crítica y reflexiva de los artículos, así como de los resultados convergentes o divergentes en relación a la investigación. Los datos extraídos fueron transcritos en un cuadro que agrupa la información de la siguiente manera: la base de datos, título del artículo, año, diseño del estudio, país, población. La exclusión de algunos artículos correspondió a que se centraban en el estudiante y no en el docente, como por ejemplo el modo de aprender de los estudiantes, o las diferentes formas de aprendizaje.

\section{Interpretación de resultados.}

Los resultados fueron presentados en forma descriptiva, en tres etapas. En la primera se describió los datos de identificación de las publicaciones (autores/as, año), la base de datos, título del artículo, diseño del estudio, país, población el país donde se realizó el estudio y el tipo de revista científica. En la segunda, se evaluaron las características metodológicas del estudio; y en la tercera etapa se describieron los resultados obtenidos en cuanto a las características docente y su importancia en el personal que ejerce la enseñanza. Para esto los resultados fueron agrupados en 2 categorías: docencia a nivel universitario, innovación en docencia.

6. Presentación de la revisión (Melo, Barbosa \& de Souza, 2011). Según ésta incluye un análisis amplio de investigaciones relevantes que dan soporte a la toma decisiones.

\section{Consideraciones éticas}

Lo que se consideró al momento de realizar la revisión de los artículos fue respetar los derechos de autor, lo cual se evidencia al citar a los autores de cada trabajo.

\section{RESULTADOS}

En esta revisión de literatura fueron incluidos siete artículos (véase tabla 1). 
TABLA 1. Universidad de Costa Rica. Distribución de las publicaciones sobre características del docente universitario (2014)

\begin{tabular}{|c|c|c|c|c|c|c|}
\hline $\begin{array}{c}\text { Base de } \\
\text { datos }\end{array}$ & Título del artículo & Año & País & $\begin{array}{l}\text { Diseño del } \\
\text { estudio }\end{array}$ & Población & Observaciones \\
\hline EBSCO & $\begin{array}{l}\text { La evaluación de la } \\
\text { actividad docente de la } \\
\text { Escuela de Enfermería de } \\
\text { la Universidad de Costa } \\
\text { Rica. }\end{array}$ & 2007 & $\begin{array}{l}\text { Costa } \\
\text { Rica }\end{array}$ & $\begin{array}{l}\text { Cualitativa } \\
\text { fenomenológica. }\end{array}$ & $\begin{array}{l}3 \text { Ex Directoras. } \\
1 \text { Directora. } \\
\text { 25 Docente } \\
\text { Régimen } \\
\text { Académico. } \\
23 \text { Docente } \\
\text { Clínicos. } \\
36 \text { estudiantes de } \\
\text { último año. }\end{array}$ & $\begin{array}{l}\text { Artículo basado en investigación que se } \\
\text { realiza para conocer las maneras en que } \\
\text { se evalúan las características y } \\
\text { actividades docentes, y si los } \\
\text { instrumentos utilizados evalúan no solo } \\
\text { la teoría, sino también la práctica } \\
\text { docente como tal. }\end{array}$ \\
\hline $\begin{array}{l}\text { Directory of } \\
\text { Open } \\
\text { Access } \\
\text { Journals }\end{array}$ & $\begin{array}{l}\text { Mediadores y mediadoras } \\
\text { del aprendizaje. } \\
\text { Competencias docentes en } \\
\text { los entornos virtuales de } \\
\text { aprendizaje. } \\
\end{array}$ & 2012 & España & $\begin{array}{l}\text { Cuantitativo, } \\
\text { descriptivo. }\end{array}$ & $\begin{array}{l}19075 \text { estudiantes } \\
\text { de } 1366 \text { cursos de } \\
\text { teleformación. }\end{array}$ & $\begin{array}{l}\text { Artículo basado en investigación que se } \\
\text { realiza en busca de identificar las } \\
\text { competencias docentes necesarias para } \\
\text { desarrollar entornos virtuales de } \\
\text { aprendizaje. }\end{array}$ \\
\hline EBSCO & $\begin{array}{l}\text { Estilos de docencia y } \\
\text { evaluación de los } \\
\text { profesores universitarios y } \\
\text { su influencia sobre los } \\
\text { modos de aprender de sus } \\
\text { estudiantes. }\end{array}$ & 2008 & España & $\begin{array}{l}\text { Cuantitativo, } \\
\text { cuasi } \\
\text { experimental. }\end{array}$ & $\begin{array}{l}42 \text { Profesores. } \\
753 \text { Estudiantes. }\end{array}$ & $\begin{array}{l}\text { Articulo basado en investigación que } \\
\text { busca analizar el cómo los modos de } \\
\text { enseñar influyen en las maneras de } \\
\text { aprender de los estudiantes. }\end{array}$ \\
\hline EBSCO & $\begin{array}{l}\text { Concepción de Estudiantes } \\
\text { y Docentes del buen } \\
\text { profesor universitario. }\end{array}$ & 2010 & Chile & $\begin{array}{l}\text { Cualitativo } \\
\text { exploratorio. }\end{array}$ & $\begin{array}{l}293 \text { estudiantes, } \\
\text { último año } \\
\text { curricular. } \\
104 \text { docentes } \\
\text { diferentes } \\
\text { profesiones. }\end{array}$ & $\begin{array}{l}\text { Artículo basado en investigación que } \\
\text { tiene como objetivo conocer la } \\
\text { representación que tanto estudiantes } \\
\text { como docentes, tiene del buen profesor } \\
\text { universitario. }\end{array}$ \\
\hline EBSCO & $\begin{array}{l}\text { Saberes docentes y } \\
\text { enseñanza universitaria. }\end{array}$ & 2012 & España & $\begin{array}{l}\text { Cualitativo } \\
\text { descriptivo. }\end{array}$ & $\begin{array}{l}90 \text { profesores } \\
\text { experimentados. } \\
11 \text { profesores con } \\
\text { menos de } 5 \text { años. } \\
301 \text { estudiantes. } \\
\end{array}$ & $\begin{array}{l}\text { Artículo que presenta los resultados de } \\
\text { una investigación cuyo objetivo fue } \\
\text { exponer la complejidad de la enseñanza } \\
\text { universitaria. }\end{array}$ \\
\hline EBSCO & $\begin{array}{l}\text { El perfil profesional de los } \\
\text { formadores de } \\
\text { Formación continua en } \\
\text { España. }\end{array}$ & 2011 & España. & $\begin{array}{l}\text { Cuantitativo } \\
\text { descriptivo. }\end{array}$ & 606 Docentes. & $\begin{array}{l}\text { Artículo que analiza y describe los } \\
\text { perfiles de los formadores en España, } \\
\text { siendo la variable experiencia } \\
\text { ocupacional una de las más relevantes en } \\
\text { el docente. }\end{array}$ \\
\hline EBSCO & $\begin{array}{l}\text { ¿Cómo es el buen profesor } \\
\text { universitario según el } \\
\text { alumnado? }\end{array}$ & 2010 & España & $\begin{array}{l}\text { Cualitativo } \\
\text { descriptivo. }\end{array}$ & 125 estudiantes & $\begin{array}{l}\text { Artículo basado en una investigación } \\
\text { cuyo objetivo es la construcción del } \\
\text { concepto del buen profesor universitario } \\
\text { desde la prospectiva del estudiante. }\end{array}$ \\
\hline
\end{tabular}

Fuente: elaboración propia.

Para mejor comprensión de las características docentes, se reunieron los resultados de los artículos incluidos en dos categorías temáticas, a saber: docencia a nivel universitaria (cinco artículos), e innovación en docencia (dos artículo).

\section{Docencia a nivel universitaria.}

La docencia universitaria tiene características muy diferentes a la docencia que se imparte en otros niveles educativos. Esto puede ser debido a que los profesores tienen una formación y expertisia en su formación profesional, no así en "educación” específicamente. 


\section{Revista Electrónica Enfermeria Actual en costa Rica}

\section{Innovación en docencia.}

El y la docente universitaria debe utilizar técnicas innovadoras para la enseñanza y aprendizaje; sin embargo, la evidencia recuperada en muy pocos casos hace mención específica al docente en Enfermería.

\section{DISCUSIÓN}

Se ha demostrado que el o la docente universitario, además de ser expertos en su campo, se debe involucrar con diversos aspectos del estudiantado como son: creencias, valores y actitudes para lograr ser guía y acompañante durante el proceso educativo, por lo que debe tener ciertas habilidades y preparación en el área de la docencia para lograr los objetivos planteados e ir más allá de un simple trasmisor conocimiento (Francis, 2005).

Relacionado con lo anterior, bajo el concepto de la "buena enseñanza", Jarauta (2012) mostró la complejidad del conocimiento profesional para la enseñanza universitaria en cuanto a su estructura, origen y formas de manifestarse en la enseñanza presencial, haciéndola diferente a las demás enseñanzas, como es el ejemplo de la primaria o la enseñanza secundaria.

Si se considera al docente como una parte clave de la una adecuada educación, Casero (2010), en busca de cómo debe ser el buen profesor universitario según el estudiantado, concluyó que éstos mencionan dos dimensiones que debe tener el docente: una como profesional y experto en su campo respectivo (saber y saber hacer), y otra como persona y su relación con los estudiantes (saber ser). Esto lo apoya Martín (2008) que en una investigación concluye que el éxito de la práctica docente está en su preparación técnica y metodológica, ya que logra en el estudiante un aprendizaje activo y efectivo. De esta manera, se refuerza la idea que el docente universitario debe contar además de su dominio en su campo profesional, con una variedad de características y conocimientos académicos, para poder desarrollar las estrategias necesarias que garanticen un adecuado proceso de enseñanza-aprendizaje.

Por lo anterior, es importante destacar que dentro del perfil profesional del docente debe destacarse la formación continua, según concluyó Mamaqi (2012), ya que de no ser así, se daría una deficiencia en la formación del estudiante. El campo disciplinar y pedagógico, no pueden ser independientes uno de otro, ya que deben interactuar para poder llegar hacer adecuada lectura de la realidad, desde sus diferentes enfoques, además se debe considerar que los cambios actuales no afectan a una disciplina aislada, sino que enfrentamos un cambio global en la concepción del saber.

Respecto de la innovación, los entornos virtuales resultan una buena opción para la enseñanza, según Menédez (2012)

si la figura docente ha ido cambiando a lo largo de los siglos con la modificación de las condiciones de los procesos educativos, se hace aun más evidente el desarrollo de la enseñanza en los nuevos entornos virtuales, que requieren una mediación del aprendizaje distinta a los entornos presenciales. (p. 49).

Lo anterior fuerza al docente a mejorar su preparación técnica y metodológica. 


\section{Revista Electrónica Enfermeria Actual en costa Rica}

Cabalín (2010) menciona que para lograr aplicar la innovación educativa "es necesario que el docente universitario modifique sus prácticas pedagógicas con los estudiantes, con una mayor interacción interpersonal o humanización.” (p.289). Además recalca que las prácticas pedagógicas que el docente utilice deben buscar una mayor interacción con sus estudiantes, por lo que si este docente no tiene conocimiento de las mismas, nunca logrará este objetivo.

De esta manera, se observa que el tema sobre las características que debe tener todo profesor universitario, se ha tocado de diversas maneras. Específicamente a la enseñanza de la enfermería, Peña (2003) menciona que se requiere un docente de enfermería que posea ciertas características que le permitan no solo constituirse en facilitador de aprendizaje, sino lograr desarrollar en el estudiante sensibilidad social, humanismo y valores.

Aguirre (2007), determinó las fallas o debilidades como docentes de enfermería dado que impera una dificultad en la enseñanza. Entre las deficiencias mencionadas está la falta de educación continua sobre didáctica, pedagogía y enseñanza, ya que solamente se desarrolla un curso de didáctica como obligación para ingresar a Régimen Académico ${ }^{1}$.

En la Universidad de Costa Rica como política académica, se realizó una investigación por medio de tres fases; la primera fase incluyó el proceso de planificación, ejecución y sistematización del taller denominado "La construcción del perfil para el profesor de la Universidad de Costa Rica", para que a partir de reflexiones realizadas por académicos y académicas determinar el perfil del profesorado. En la segunda fase, se dedicaron a revisar, analizar y sistematizar literatura acerca de retos y desafíos de las universidades públicas en el nuevo milenio, nuevas tendencias en la formación del profesorado universitario, perfil del docente universitario, y competencias laborales. Finalmente, en la tercera fase, mediante sesiones de trabajo, se determinaron y evaluaron las competencias que requiere el profesorado de la institución.

Como resultado de esta investigación se obtuvo un perfil de competencias para el profesorado de la Universidad de Costa Rica según se establece en el acta de la sesión N. ${ }^{\circ} 4926$ del Consejo Universitario celebrada el miércoles 27 de octubre del 2004, donde se establecen cinco aéreas como requisito que debe cumplir el docente universitario: docencia, investigación, acción social, personal y ético institucional.

Relacionado con lo anterior, en abril del año 2015, se realizó la "Primer Conferencia Nacional sobre modelo de atención y formación de profesionales en ciencias de la salud en Costa Rica", la cual entre sus objetivos, estuvo "Identificar los mecanismos idóneos para garantizar la calidad y pertinencia de la formación de profesionales en ciencias de la salud, en relación con las necesidades de salud de la población" (Informe Final, 2015).

Entre otros temas, se discutió sobre si se busca formar un recurso humano la calidad, el personal que busca ese recurso humano también debe ser de calidad (Informe final, 2015). Se hizo referencia al igual que se ha mencionado anteriormente, que el personal docente que forma estos profesionales en salud no debe ser solo

\footnotetext{
${ }^{1}$ El Régimen Académico de la Universidad de Costa Rica es el sistema que organiza a los profesores universitarios en categorías con base en sus méritos académicos y en su experiencia universitaria. Tomado del REGLAMENTO DE RÉGIMEN ACADÉMICO Y SERVICIO DOCENTE (Aprobado en sesión 2869-17, 16/02/1982. Publicado en Gaceta Oficial 76-82, 22/04/1982)
} 


\section{Revista Electrónica Enfermeria Actual en costa Rica}

capaz en su campo disciplinar, sino que este debe estar formado en docencia, "conocedor de las ciencias pedagógicas para que realmente sepa cómo enseñar" (p.143).

Entre la discusión en sub plenarias, se llego a la conclusión que los encargados de formar a los futuros profesionales en salud debe ser "un cuerpo docente que sea capaz de adaptarse a las características de los distintos grupos estudiantiles" (p.143), ya que por falta de conocimiento en este campo la mayoría de los profesionales que se dedican a la docencia universitaria, lo que hacen es un réplica de cómo le enseñaron sus antecesores.

Por lo tanto, y con base a estas necesidades discutidas en esta primera conferencia, también se discutió el tema de los procesos de acreditación, los cuales se considera de importancia que tomen el tema de la docencia como un criterio de cumplimiento (Informe final, 2015), donde se busque una mayor rigurosidad en los procesos de selección para la contratación de personal docente, ya que "hoy en día es práctica común que se nombren como docentes a estudiantes recién graduados... nada más porque tuvieron buen desempeño en su fase de estudiante" (p.143).

Específicamente en el ámbito de la enfermería, esta preocupación por la manera en que se forman los futuros profesionales en este campo, radica desde 1917 cuando se organizó el primer programa de enfermería dependiente de la Facultad de Medicina, el cual buscó la mejora en la calidad de la enseñanza. Por medio de Sor Ángela Lazo, supervisora de las hermanas de la caridad del Hospital San Juan de Dios, directora del servicio de enfermería y directora de la Escuela de Enfermería en ese momento, se introdujeron materias nuevas y se equipó el primer laboratorio de enfermería para la práctica de las estudiantes. (Mora-Quirós y Caravaca-Morera, 2014)

Bajo esta misma línea, en 1951 se firmó un convenio con la Organización Panamericana de la Salud (OPS) y la Organización Mundial de la Salud (OMS), donde se establecía un programa de becas para preparar enfermeras nacionales en pedagogía en enfermería y especialidades en algún área de enfermería (Mora-Quirós y Caravaca-Morera, 2014), evidenciado de esta manera la importancia que se ha dado en la formación de docentes en enfermería.

En 1953, comenzó a funcionar el primer grupo de enfermeras nacionales especialmente preparadas para la enseñanza de enfermería que habían realizado sus estudios en universidades extranjeras, desempeñándose como profesoras de la Escuela de Enfermería. (Mora-Quirós y Caravaca-Morera, 2014) Después de esto, se continúo con la preparación de enfermeras para la especialización en la enseñanza de diversos campos de la enfermería, por medio de becas otorgadas por la Organización Mundial de la Salud.

Con forme avanzaron los años y se fue progresando en el tema de la enseñanza en la enfermería, en 1975 por acuerdo del Consejo Universitario, se aprobó la incorporación definitiva de la Escuela de Enfermería a la Universidad de Enfermería.

En la actualidad, la enfermería al estar inmersa dentro de los procesos de cambio y actualización curricular y pedagógico, ha buscado nuevos enfoques de atención, basados en un campo de acción amplio, bajo un paradigma transformador, con el fin de que, por medio de sus estudiantes, se llegue a brindar una atención integral a los usuarios y usuarias, así como un acompañamiento continuo a los mismos en su proceso de salud enfermedad. 


\section{Revista Electrónica Enfermeria Actual en costa Rica}

En busca de nuevos enfoques, en 1997 la Escuela de Enfermería realizó una revisión del plan de estudios y se creó un nuevo plan, según la Resolución número VD-6479-98, en el cual la y el estudiante se visualiza como persona participativa de su proceso de construcción de conocimiento, para que desde sus inicios llegue a desarrollar una visión de enfermería dinámica, actualizada y empoderada de su conocimiento, tratando todos los aspectos que influyen en un individuo, con el fin de trabajar con ellos en su autocuidado, coincidiendo esto el propósito de la unidad académica: "Formar profesionales en enfermería en el nivel de grado y posgrado, con capacidad crítica y analítica, poseedora de libre pensamiento...". (Escuela de Enfermería. Universidad de Costa Rica, 2013).

En el ámbito pedagógico, la Escuela de Enfermería propone un modelo constructivista, ya que este considera que el aprendizaje es un proceso complejo de construcción, basado en el conocimiento previo del estudiante (Garzón y Vivas, 1999) Esta posición rompe con las utilizadas desde décadas pasadas, donde se consideraba al docente como la fuente directa de conocimiento, el cual debía ser trasmitido al alumno en forma de clases magistrales, utilizando el método de memorización. Por tal razón y debido a que, hasta hace muy pocos años, no existía una carrera de docencia universitaria como tal, se dejaba de lado la enseña en docencia, como algo empírico, donde los nuevos profesores deben repetir comportamientos aprendidos de sus mismos antecesores, cuando eran estudiantes.

De esta manera, para poder llegar a romper estos paradigmas y en busca del cumplimiento de las metas y objetivos pedagógicos propuestos por la Escuela de Enfermería, los nuevos docentes deben aprender estrategias didácticas, tomando en cuenta los nuevos modelos pedagógicos, planteando a la población estudiantil interrogantes a partir de pre concepciones, con el fin de incitar a buscar y construir conceptos que permitan dar un significado más complejo, que lo expuesto por el mismo profesor(a). De tal manera, que el docente llegue a ser un mediador en el proceso de enseñanza y aprendizaje, teniendo claro que cada estudiante es un individuo independiente, único y responsable de generar su propio conocimiento, por lo que no puede tomar una actitud pasiva, sino más bien desarrollar actividades creativas y productivas. (Zabalza-Beraza, 2007)

Entre los aportes que los cambios pueden producir, se encuentra el hecho de lograr una interacción establecida entre el sujeto y el objeto del conocimiento, pasando de una educación centrada en la trasmisión de conocimientos, a otra que, integrando la investigación se dedique a crear saberes y nuevas prácticas, buscando una interacción entre lo cognitivo y lo vivencial.

Por lo que la enseñanza de enfermería requiere docentes cuyo compromiso sea promover la participación activa, por medio de la construcción de conocimiento, preparando al nuevo profesional, para enfrentar los retos futuros, siendo capaz de aplicar estrategias orientadas a la transformación de su realidad. (Peña, 2013).

Además, Peña (2013) considera que el profesional en enfermería que practica la docencia, no sólo debe mantener actualizados sus conocimientos en docencia, también debe conocer metodología en investigación, así como saber trasmitir por medio del ejemplo el modelo profesional, ser puntual con los usuarios y los estudiantes, ser veraz para aclarar las dudas del estudiante durante las clases teóricas o prácticas, tratando respetuosamente al estudiante y escuchado con atención al usuario. 


\section{Revista Electrónica Enfermeria Actual en costa Rica}

Adicionalmente a las recomendaciones ya realizadas, los profesionales de enfermería dedicados a la docencia deben tener presente que su práctica docente es algo construido en el día a día, donde el profesor cumple el papel de receptor y dador de información a través de diferentes medios, como lo son los gestos, las palabras, los métodos de enseñanza, la flexibilidad y rigidez en el proceso de aprendizaje, que sirve de base a la conducta de los estudiantes, así como a la de los egresados. (Palencia, 2006).

\section{CONCLUSIONES}

La mayoría de los autores coinciden que la selección de los docentes universitarios y su proceso de desarrollo académico no puede limitarse a lo disciplinar, deberá asumir lo personal y lo pedagógico, de hecho lo pedagógico no existe si no es al interior de una disciplina. Además, de ser experto en el campo que enseña es desarrollador de investigación, acción social.

Aunque existe un perfil de competencias establecidas para el docente en Universidad de Costa Rica, no existe un perfil específico que determine cuales son las características o cualidades que se requiere el docente de enfermería en la Escuela de Enfermería.

Hay carencia de investigación respecto de las competencias que debe tener una enfermero (a) que se dedique a la docencia en la Escuela de Enfermería de la Universidad de Costa Rica específicamente.

\section{Conflicto de intereses} financiero.

El autor declara que no existe conflicto de intereses de orden personal, comercial, académico, político o

\section{REFERENCIAS BIBLIOGRÁFICAS}

Aguirre, E. (2007). La evaluación de la actividad docente de la Escuela de Enfermería de la Universidad de Costa Rica. Revista Educación 31(1), 65-76. Recuperado de: www.redalyc.org/articulo.oa?id=44031104

Bain, K. (2006). Lo que hacen los mejores profesores universitarios. Valencia: PUV. Broome, ME. (2000) Integrative literature reviews for the development of concepts. In: Rodgers BL, Knafl KA, organizador. Concept development in nursing: foundations, techniques and applications. Philadelphia: W.B. Saunders Company; p.231-50.

Broome, ME. (2000) Integrative literature reviews for the development of concepts. In: Rodgers BL, Knafl KA, organizador. Concept development in nursing: foundations, techniques and applications. Philadelphia: W.B. Saunders Company; p.231-50.

Cabalín, S. (2010). Concepción de Estudiantes y Docentes del buen profesor universitario. Int. J. Morphol., 28(1), 283-290. Recuperado de: $\underline{w w w . s c i e l o . c l / s c i e l o . p h p ? s c r i p t=s c i ~ a r t t e x t \& p i d=S 0717-95022010000100042 ~}$ 


\section{Revista Electrónica Enfermeria Actual en costa Rica}

Casero-Martínez, A. (2010). ¿Cómo es el buen profesor universitario según el alumnado? Revista española de pedagogía año LXVIII, 246, 223-242. Recuperado de: https://dialnet.unirioja.es/descarga/articulo/3206367.pdf

Canalejas-Pérez, $M^{a}$., Martínez-Martín, $M^{\mathrm{a}}$., Pineda-Ginés, Mª., Vera-Cortés, M., Soto-González, M., MartínMarino, A. y Cid-Galán, $M^{a}$. (2005). Estilos de aprendizaje en los estudiantes de enfermería. Escuela Universitaria de Enfermería La Paz, La Paz.

Consejo Universitario (2004). Perfil de competencias para el profesorado de la UCR. Sesión 4926. Universidad de Costa Rica. Escuela de Enfermería. (1997). Restructuración del bachillerato que se transforma en la licenciatura en enfermería. Resolución N 6479-98. Tomo I y II. Universidad de Costa Rica.

Escuela de Enfermería. (1997). Restructuración del bachillerato que se transforma en la licenciatura en enfermería. Resolución N 6479-98. Tomo I y II. Universidad de Costa Rica.

Facultad de Medicina (2015) Primera Conferencia Nacional sobre modelos de atención y formación de profesionales en ciencias de la salud en Costa Rica. Universidad de Costa Rica.

Francis, S. (2005). El conocimiento pedagógico del contenido como categoría de estudio de la formación docente. Actualidades investigativas en educación. INIE-UCR. Recuperado de: www.redalyc.org/articulo.oa?id=44750211

Francis, S. (2010). Hacia una caracterización del docente universitario excelente. Revista Electrónica Investigación Educación 30 (1). Recuperado de: www.revistas.ucr.ac.cr/index.php/educacion/article/download/1793/1766

Garzon, C., Vivas, M. (1999). Una didáctica constructivista en el aula universitaria. EDUCERE, ARBITRADA, AÑO 3, No 5. Recuperado de: http://www.saber.ula.ve/bitstream/123456789/19454/1/articulo3-5-5.pdf

Ganong, LH. (1987). Integrative reviews of nursing research. Res Nurs Health, 10(1),1-11.

Jarauta-Borrasca, B. y Medina-Moya, J. (2012). Saberes docentes y enseñanza universitaria. Estudios sobre educación. 22, 179-198. Recuperado de: https://www.unav.edu/publicaciones/revistas/index.php/estudiossobre-educacion/article/view/2078

Mamaqi, X. y Miguel, J. (2011). El perfil profesional de los formadores de formación continúa en España. RELIEVE, 17 (1), 1-32. Recuperado de: http://www.uv.es/RELIEVE/v17n1/RELIEVEv17n1_2.htm

Martin, D., Soler, A., Broche, R., Alonso, Z. y Vigo, P. (2008) Caracterización de la práctica docente en Morfofisiología Humana I y II. Educ Med Super [online]., 22 (4). Recuperado de: http://scielo.sld.cu/scielo.php?script=sci_arttext\&pid=S0864-21412008000400002 


\section{Revista Electrónica Enfermeria Actual en costa Rica}

Mata-Terrón, A., Tójar-Hurtado, J. y Serrano-Angulo, J. (2004). Innovación educativa: un estudio de los cambios diferenciales entre el profesorado de la Universidad de Málaga. Revista Electrónica de Investigación Educativa 6 (1). Recuperado de: http://redie.uabc.mx/redie/article/viewFile/94/163

Medina, J. (1999). La pedagogía del cuidado: Saberes y prácticas en la formación universitaria en enfermería. Barcelona: Editorial Laertes.

Melo, M. B., Barbosa, M. A., \& de Souza, P. R. (2011). Satisfacción en el trabajo del equipo de enfermería: revisión integradora. Rev. Latino-Am. Enfermagem 19(4), 1-10. Recuperado de: http://www.scielo.br/scielo.php?pid=S0104-11692011000400026\&script=sci_abstract\&tlng=es

Menéndez, C. (2012). Mediadores y mediadoras del aprendizaje. Competencias docentes en los entornos virtuales de aprendizaje. Revista Iberoamericana de Educación. 60, $39-50$ (1022-6508) Recuperado de: www.rieoei.org/rie60a02.pdf

Mendes, K., Silveira, R. C. y Galvão, C.M. (2008). Revisão integrativa: método de pesquisa para a incorporação de evidências na saúde e na enfermagem. Texto \& Contexto - Enfermagem, 17(4), 758-764.

Mora-Quirós, S. y Caravaca-Morera, J. (2014). Enfermería en Costa Rica: Una Mirada Histórica. (AU) / Nursing in Costa Rica: an historical view. Hist. enferm., Rev. eletronica;5(2):280-297, ago.-dez. 2014. Recuperado de: http://www.here.abennacional.org.br/here/vol5num2artigo9.pdf

Palencia, E. (2006). Reflexión sobre el ejercicio docente en nuestros días. Universidad de Antioquia, Facultad de Enfermería. Medillín-Colombia.

Peña, L.(2003) Características requeridas en el docente de enfermería según la opinión de la comunidad educativa de enfermería de la UNMSM 2001-2001. Tesis para optar por el título profesional de Licenciada en Enfermería. UNMSM. Lima-Perú.

Piedra-González, M., Solano-González, A., Villalobos-Solano, L. (2015). Primera conferencia nacional sobre modelo de atención y formación de profesionales en ciencias de la salud en Costa Rica. Informe Final. Facultad de Medicina. Universidad de Costa Rica.

Preciado-Cortes, F. (2008). Ser y quehacer docente en la última década. RMIE,13 (39), 1139-1163. Recuperado de: http://www.redalyc.org/pdf/140/14003906.pdf

Suares-Suares, G., Iglesias-León, M. y Basulto-Marrero, B. (2012). La formación y desarrollo basado en competencias de los profesores universitarios en la Filial de Aguada de Pasajeros. Pedagogía Universitaria. Vol. XVII (2). Recuperado de: www.cvi.mes.edu.cu/peduniv/index.php/peduniv/article/download/24/24

Viale, H. (2011). Organización de la clase: ¿preparo mi clase para enseñar o para que el alumno aprenda?. Revista Digital de Investigación en Docencia Universitaria / Año 5,(1). Recuperado de:

http://www3.upc.edu.pe/bolsongei/bol/16/775/Articulo-Organizacion-de-la-clase-Hector-Viale.pdf 


\section{Revista Electrónica Enfermeria Actual en costa Rica}

Whittemore R, Knafl K. (2005). The integrative review: updated methodology. J Adv Nurs, 52(5):546-53.

Zabalza-Beraza, M. (2007). La didáctica universitaria. Bordón. Universidad Santiago de Compostela. Revista de pedagogía, ISSN 0210-5934, ISSN-e 2340-6577, Vol. 59, № 2-3, 2007, págs. 489-510. Recuperado de: file:///C:/Documents\%20and\%20Settings/Derby/Mis\%20documentos/Downloads/Dialnet-

LaDidacticaUniversitaria-2553099.pdf 